\title{
La norma jurídica referida a la atención de la niñez menor de tres años en Costa Rica, ¿un verdadero respaldo o una utopía?
}

\author{
The juridical norm recounted for the attention of the childhood three-year-old minor in Costa Rica, \\ a real support or a Utopia?
}

Helvetia Cárdenas Leitón ${ }^{1}$

Recibido: 27/8/2015 / Aprobado: 12/4/2016

\begin{abstract}
Resumen
Este artículo se deriva de un proceso de investigación sobre La educación y atención integral para el desarrollo de la niñez menor de 3 años en Costa Rica. Discute sobre las características que presenta la documentación legal que vincula la atención de la niñez menor de tres años en Costa Rica. El acercamiento al tema se realizó desde el paradigma cualitativo y el método de análisis elegido fue el hermenéutico-dialéctico, el cual permitió profundizar en algunos documentos, así como interpretar el discurso de las personas participantes. Se utilizó el análisis documental de diferentes fuentes bibliográficas; entre ellas, las propuestas programáticas existentes en el país para la atención de este grupo poblacional, la Política nacional para la niñez y la adolescencia y el Código de la niñez y la adolescencia, se empleó también la entrevista en profundidad y grupos focales con especialistas en diversas áreas relacionadas con esta población. Entre las conclusiones obtenidas se evidencia que la legislación en sí misma no garantiza su adecuada aplicación y que a pesar de la existencia de dicho marco legal, el Estado costarricense no ha brindado una respuesta efectiva para la educación integral de la niñez menor de tres años, razón por la cual es necesario identificar mecanismos efectivos para su cumplimiento.
\end{abstract}

Palabras clave: legislación para la infancia, primera infancia, atención integral, política educativa de niñez.

\section{Abstract}

This article is derived from a research study on Education and comprehensive care for the development of children under 3 years in Costa Rica. It delves into the characteristics included in legal documentation linked to care for children under 3 in Costa Rica. This investigation followed a qualitative paradigm. A dialectic-hermeneutical method of analysis was implemented which allowed to examine selected documents and interpret the discourse of participants. Document analyses of different bibliographical sources were performed, which include among others current program proposals in the country to assist this population, State Policies for Children and Adolescents, and Children and Adolescents Regulations. In-depth interviews and focus groups with specialists in diverse areas that have affiliations with this population were implemented. Among the conclusions drawn, there is evidence to claim that the legislation itself does not guarantee its proper application, and despite the existence of this legal framework, the Costa Rican government has failed to provide comprehensive education for children under 3 years. Thus, it is necessary to identify effective mechanisms to guarantee compliance.

Keywords: Legal framework, early childhood, Costa Rica, utopia, policies.

${ }^{1}$ Doctora en Educación, Máster en Psicopedagogía, Licenciada en Administración Educativa y Educación Preescolar, Universidades CONARE. Directora del Recinto de Grecia y Subdirectora del Instituto de Investigación en Educación Universidad de Costa Rica. Correo electrónico: hcardena19@gmail.com 


\section{Introducción}

El marco legal vinculado con la atención de la niñez menor de tres años en Costa Rica se corresponde con a los lineamientos y principios de la UNICEF, pues tanto la Política nacional para la niñez y la adolescencia (2009) como el Código de la niñez y la adolescencia (1998) contienen orientaciones posicionadas en acuerdos internacionales para la protección, equidad e igualdad de oportunidades de la niñez y la adolescencia, aspecto medular en el presente análisis, pues se partió del hecho de que la atención y educación integral es un derecho de la niñez, tal como lo fundamenta la OIT (2012), en el siglo $\mathrm{XX}$, los niños empezaron a ser percibidos como titulares de derechos y se consignan las intenciones de la Convención sobre los Derechos del Niño, tal como lo presenta la siguiente cita:

En 1989, las Naciones Unidas adoptaron la Convención sobre los derechos del niño (CDN), texto jurídicamente vinculante que constituye el tratado de derechos humanos con mayor número de ratificaciones en el mundo. En la CDN se hace hincapié en el derecho de todos los infantes a la educción y se exhorta a los gobiernos a prestar asistencia a los padres o a los representantes legales para el desempeño de sus funciones en lo que respecta a la crianza del niño, a poner a disposición servicios e instalaciones de guarda de niños, en especial para los menores cuyos padres trabajan. La CDN ha contribuido a configurar políticas y disposiciones en materia de educación de la primera infancia en todo el mundo, aunque su influencia en las decisiones en materia de gasto público de muchos gobiernos no ha sido suficiente como para garantizar el respeto efectivo de los derechos de los niños. (OIT, 2012, p.8).

A partir de esa intención, la Educación es uno de los derechos impostergables e inalienables por los cuales todas las ciudadanas y ciudadanos del país deben asumir responsabilidades y -por endecompromiso con la niñez. Al respecto (UNICEF, 2001) indica que:
El cumplimiento de los derechos de la niñez y la adolescencia es responsabilidad de todos los sectores: del Estado y de la sociedad civil, $y$ es consecuencia de las interrelaciones que se logren establecer entre ellos a través de la legislación (p.23).

De lo anterior se infiere que existe mayor conciencia de los derechos de la niñez y se esperaría con ello una garantía de su cumplimiento. A partir de esa premisa la educación y atención de la niñez menor de tres años, corresponde además con un derecho fundamental al cual el Estado y la comunidad en general deben responder, ya que con esa pretensión, no solo se protege la integralidad de los niños, sino que se respeta su desarrollo.

La niñez - sin distingos de edad, etnia o condición social- debe recibir una educación pertinente y relevante como derecho inalienable que potencie sus facultades para su desarrollo integral. En ese sentido la educación inicial representa una respuesta educativa para el desarrollo de los niños. En consecuencia no se debe limitar a la niñez cuyos padres trabajan quienes requieren apoyo para el cuido de sus hijas e hijos. Por el contrario, la educación y atención se deben asumir como un derecho que tiene todo niño a poder participar en situaciones educativas distintas a las que se producen en la familia y que -por supuesto- promueven su desarrollo.

Se considera oportuno referirse a la opinión de Evans, Myers e Ilfeld, citados por (Chavarría et al. 2007), con respecto a lo que implica un enfoque de derechos, pues esta premisa convoca a repensar de manera crítica si en realidad la atención de la niñez en los espacios dispuestos para ello, responden más a satisfacer sus necesidades básicas que a potenciar su desarrollo integral. Al respecto los autores, señalan que:

Un enfoque de derechos entraña un imperativo de atención universal, mientras que un enfoque orientado a las necesidades puede incluir metas parciales. A la vez, los derechos no pueden organizarse jerárquicamente y guardan relación con una obligación y no con un sentido de caridad. (p. 97) 
Esta posición exigiría un abordaje más apegado a la atención y a la educación como derechos inalienables, de tal forma, que las políticas destinadas para la niñez, trascenderían los espacios hacia propuestas curriculares más holistas y críticas que garantizarán un desarrollo óptimo de ese grupo poblacional.

\section{El marco jurídico internacional}

En el afán de poder cumplir a cabalidad con la atención y educación de la niñez, el Estado costarricense ha ratificado una serie de convenios internacionales que complementan la normativa nacional existente, los cuales se presentan en la Tabla 1:

\section{Tabla 1: Marco jurídico internacional que protege a la niñez}

\begin{tabular}{ll}
\hline 1924 & $\begin{array}{l}\text { "Declaración de Ginebra sobre los derechos del niño", adoptada en } 1948 \text { en la Asamblea } \\
\text { General de las Naciones Unidas. }\end{array}$ \\
\hline 1959 & "Declaración de los Derechos del Niño", Asamblea General de las Naciones Unidas. \\
\hline 1994 & Año Internacional de la Familia. \\
\hline 1995 & IV Conferencia Mundial sobre la mujer en Beijín. \\
\hline 2010 & Objetivos del Milenio \\
\hline
\end{tabular}

Fuente: Elaboración propia con datos tomados de la normativa internacional

A partir del marco jurídico internacional, el Estado costarricense al ratificar como se puede observar, los convenios internacionales, se compromete a considerar, proteger y asumir acciones en beneficio de la niñez. No obstante, debe aclararse que todas las Convenciones de las Naciones Unidas, la OEA, o cualquier otro organismo interestatal, son de acatamiento obligatorio para los países que las suscriben, sin embargo, ningún país está obligado a aceptarlas en primera instancia. Debido a ello, su atención quedó a voluntad de los países, en consecuencia con poco impacto, tal es el caso de la Declaración de Ginebra sobre los Derechos del Niño, donde se reconoce y afirma por primera vez la existencia de derechos específicos para los niños, pero ante todo, la responsabilidad de los adultos hacia ellos, se enfatiza en el bienestar del niño y se reconoce su derecho al desarrollo, asistencia, socorro y a la protección. Al respecto Calvo et al. (2012, p. 120) señalan: "dichas declaraciones y convenciones internacionales cubrían a la población infantil, pero no especificaban qué acciones concretas debían emprender los gobiernos en materia de niñez".

En la Declaración de los Derechos del Niño (1959), se plantea la educación gratuita y obligatoria en las etapas elementales. Sin embargo, Costa Rica se anticipó a esa declaratoria con un artículo de la Constitución Política de 1949 que indica: "la educación pública será organizada como un proceso integral correlacionado en sus diversos ciclos, desde la edad preescolar hasta la Universitaria”. Es importante señalar que el Artículo 78 se modificó y a partir del año 1997 se le incluyó la Educación Preescolar como obligatoria.

En cuanto a los objetivos del milenio destacan dos aspectos fundamentales: reducir la mortalidad infantil como predictor del desarrollo de las naciones y el bienestar de su población, por una parte, y mejorar la salud materna, por otro. 


\section{Marco jurídico costarricense}

A partir de lo anterior, el marco jurídico costarricense para la protección a la niñez, resguarda la integridad y derechos de la población costarricense y se resume en la Figura 1.

Figura 1: Marco jurídico internacional que protege a la niñez

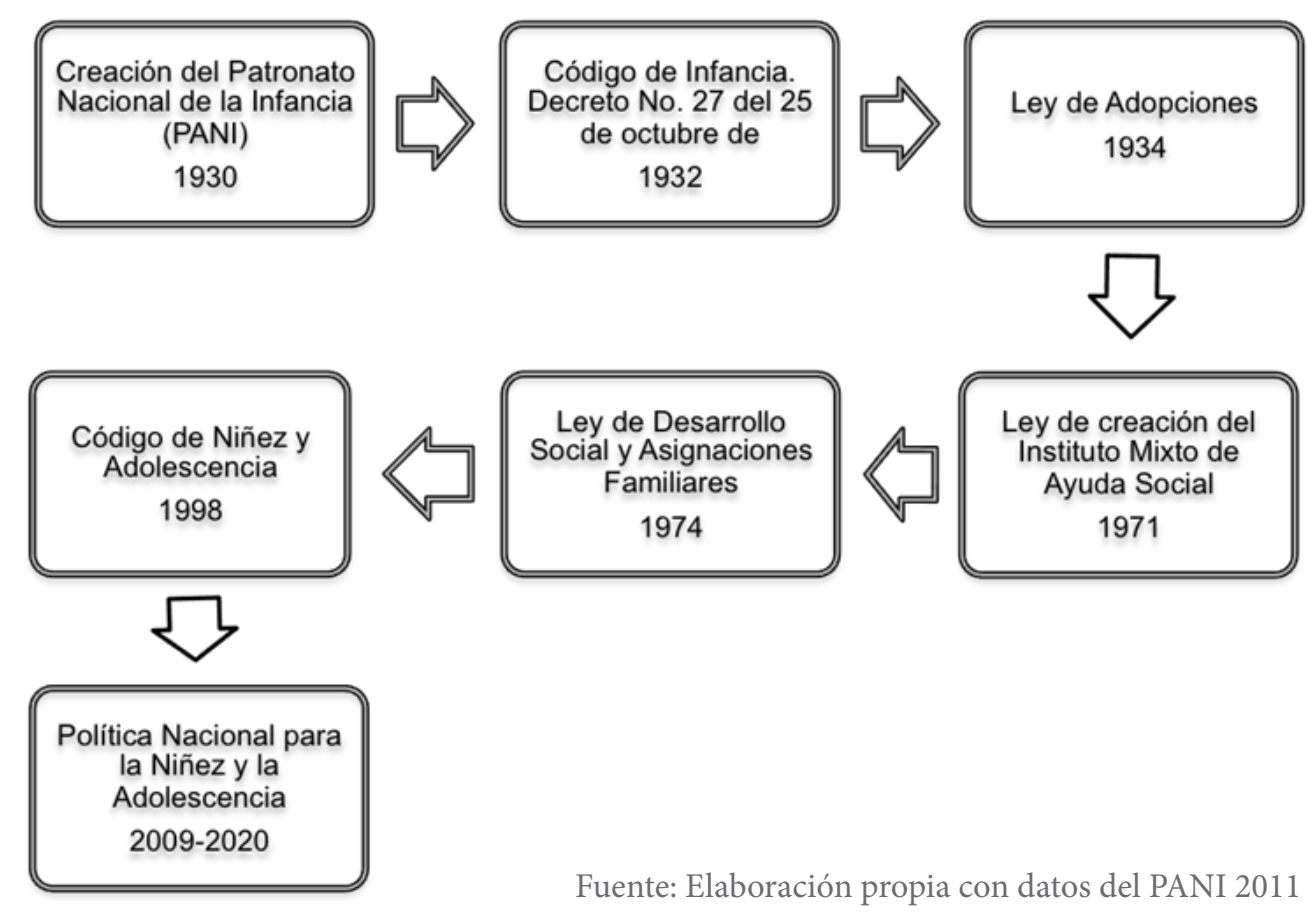

Bajo este marco, todas las acciones dirigidas a la niñez en Costa Rica deben apegarse a su cumplimiento y con el fin de orientar ese accionar el Estado creó programas para atender a la niñez menor de 6 años, unos de carácter formal, como los correspondientes al Ministerio de Educación Pública, en donde se cuenta con el nivel preescolar que involucra los ciclos de Materno infantil, el cual cubre a los niños desde los 2 meses hasta los 4 años y 6 meses; y el ciclo de Transición que atiende a la niñez con edades de 5 años y 6 meses a 6 años y 6 meses. Con respecto al ciclo Materno infantil conviene aclarar que aun cuando se especifica la atención de la niñez desde los 2 meses hasta los 4 años y 6 meses -en realidad- a partir de que el programa nació en el año 2000 atiende únicamente el grupo de Interactivo II que corresponde a la niñez de 4 años y 6 meses a 5 años y 6 meses, de manera que el resto de la población no recibe ese servicio por parte del Ministerio de Educación Pública, inclusive en el recién Programa de Estudios de Educación Preescolar 2014 (no fue considerado en el análisis realizado en la investigación), aprobado por el Consejo Superior de Educación persiste dicha política en relación con las edades.

Otros programas se enmarcan desde lo no formal y pertenecen al Ministerio de Salud, como son los Centros de Educación y Nutrición CEN (1950) y Centro de Atención Integral CINAI (1977) donde se atienden niños desde los 2 hasta los 7 años.

Por su parte, el Ministerio de Trabajo y el Instituto Mixto de Ayuda Social, tienen bajo su 
dirección otras iniciativas, aun cuando creó en el año 1991 los Hogares comunitarios, como una alternativa de alimentación, cuido, estimulación y seguridad a los niños menores de siete años en condición de pobreza.

Se recuerda, además, que existe una oferta disponible en centros infantiles de instituciones autónomas, los cuales surgen como alternativa para ofrecer educación y atención integral a la niñez menor de 5 años a través de casas infantiles o en organizaciones como el INA, ICE, INS, UCR, entre otras. También existen otros centros infantiles privados, regulados, a su vez, por el Consejo de Atención Integral (CAI) y la propuesta no convencional, como son los Jardines de párvulos de la Iglesia católica (JAPAIC) y Organización de madres maestras. Aunados a ellos están los servicios que se ofrecen en los Hospitales nacional de niños y el Calderón Guardia a través del servicio de atención a menores de tres años en escuelas para padres y otros programas. También es importante mencionar el servicio que se ofrece por parte de la reciente Red nacional de cuido y desarrollo infantil, la cual opera a través del Ministerio de bienestar social y familia, iniciativa más reciente por parte del Estado y que atiende la niñez hasta los 12 años en condición de pobreza y vulnerabilidad social.

Tal como lo indica la Ley 9220 Red nacional de cuido y desarrollo infantil (2014, p.1) en el Artículo 1.- Creación y finalidad

Se crea la Red Nacional de Cuido y Desarrollo Infantil (Redcudi), con la finalidad de establecer un sistema de cuido y desarrollo infantil de acceso público, universal y de financiamiento solidario que articule las diferentes modalidades de prestación pública y privada de servicios en materia de cuido y desarrollo infantil, para fortalecer y ampliar las alternativas de atención infantil integral.

Los servicios de la Red Nacional de Cuido y Desarrollo Infantil deberán entenderse como complementarios y no sustitutos de los servicios de educación preescolar prestados directamente por el Ministerio de Educación Pública.
Debe aclararse -además- que aparejado al surgimiento de los programas citados nace la necesidad de disponer de propuestas programáticas para orientar su accionar y es así como emergen, entre otros, los siguientes documentos:

- Guía curricular para la estimulación del desarrollo integral del niño menor de seis años del Ministerio de Salud Pública (1982).

- Serie Creciendo con amor del programa Hogares comunitarios del IMAS (1993).

- Programa Materno infantil del Ministerio de Educación Pública (2000).

- Guía de Educación inicial "Promoción de la salud en torno al eje de la infancia" (2011) del Ministerio de Salud.

- Guía para la elaboración de planes de estimulación para la promoción del desarrollo infantil de niños y niñas (sic) de 1 a 6 años y 11 meses (2011) del Ministerio de Salud.

\section{Postulados teóricos desde donde se debe posicionar la atención y educación de la niñez costarricense menor de tres años}

\subsection{Los programas para la atención de la niñez menor de tres años deben tener un enfoque holista y crítico}

La atención y educación de la niñez menor de tres años requiere una mirada transformadora, una mirada hacia experiencias educativas que le provean al niño oportunidades de todo tipo, la integración paulatina a la cultura y al mundo, es decir un desarrollo óptimo (Chavarría 2012, Peralta 2008).

A partir de esa premisa, las prácticas actuales en los espacios para la educación y la atención de los niños en los primeros años deberían de proveerle a la niñez la interacción y socialización oportunas para su integración en la cultura. Ante el escenario de incertidumbre respecto de la atención de la primera infancia, surge el holismo como una ventana de oportunidades de desarrollo para los infantes. Según a Wernicke (1994, págs.5-7): 
La palabra "holismo" proviene del griego holo, que significa entero, completo. El holismo, como movimiento educativo y terapéutico, se interesa por la interrelación entre todos los planos mencionados (físico, biológico, emocional, intelectual y espiritual) y su influencia recíproca [...] una educación holista es la que contempla al ser humano en su globalidad.

En realidad, esa es la vida, una trama de relaciones interconectadas, todo cuanto acontece en la vida de las personas las permea, las determina, las afecta positiva o negativamente. Con esa visión del acontecer, más allá de comprometerse con la integralidad, tanto las prácticas como los escenarios para la atención y educación de la niñez menor de 3 años, deben traducirse en experiencias y ambientes interrelacionados, comprometidos con la realidad, con el planeta, con su esencia, desde una visión crítica.

Tanto Peralta (2005) como Chavarría et al. (2007) realizan una comparación de orientaciones y metas desde los paradigmas tradicional y holista en Chavarría o tradicional y humanista en Peralta.

\subsection{La niñez, un estamento poblacional protegido y amado por las familias y la sociedad en general: educación y atención como derecho infantil}

En la actualidad la educación inicial ha sufrido un cambio, más y más personas son conscientes de la importancia de la atención al desarrollo integral de la niñez en edades donde tradicionalmente se dejaban únicamente a la atención de las familias, especialmente de la madre.

No puede existir discusión alguna que niegue la importancia del apego que en la niñez se desarrolla con las familias, este representa una condición determinante en la conformación del carácter, la personalidad y la sensibilidad de cada persona, es decir los adultos, de alguna manera, son el reflejo de la crianza a la cual fueron expuestos, en el buen sentido de la palabra. En la actualidad, esa función educativa y socializadora que realizaban las familias se comparte con la escuela, a la que se delegan sobre todo los aspectos más técnicos y sistemáticos en la formación de las personas.

Este trabajo está lejos de insinuar siquiera que la niñez menor de 3 años se deba institucionalizar para ser atendida por especialistas, lo que sí procura es destacar la importancia de la atención del desarrollo integral u holista en ese tiempo, como un espacio de gran trascendencia en el desarrollo humano.

Resulta interesante la discusión en relación con la atención de la niñez menor de 3 años, tal como lo señala Myers (1995) son años fundamentales en la formación de la personalidad, del apego, de los valores, las costumbres, los hábitos, para Goldschmied y Jackson (2000) "se puede juzgar a una sociedad por su actitud hacia las más pequeñas y los más pequeños, no sólo en cuanto se dice sobre ellas y ellos, sino en cómo se expresa esta actitud en lo que se les ofrece a medida que se hacen mayores", esta posición resulta congruente con lo expuesto por Da Costa (2007, p. 8) cuando indica que:

La visión postestructuralista de la infancia ha propuesto que se debe de (sic) reconsiderar la idea de lo que son los niños y niñas, la forma como ellos son educados y si la fase de la educación de la primera infancia podría evolucionar y convertirse en algo diferente basado en el cuestionamiento de la relación que se genera entre lo que conocemos y cómo usamos lo que sabemos y cómo nos posicionamos para usarle en el rol de educador.

Las ideas de poder disciplinario y de cuerpos dóciles son los lentes con los que se han examinado las prácticas en la educación preescolar. Estos cuestionamientos se basan en la gran diversidad de infancias que existen en el mundo, cada tipo de infancia está regida por el hacer social en donde tiene lugar.

Por otra parte, "la sociología de la infancia ha cuestionado las nociones "predeterminadas" (fases, estadios, periodos) sobre los niños y las niñas (sic), así mismo la idea de la "universalidad de la infancia" que promueve una forma única y particular de 
experiencia educativa dictada por estados y fases sin considerar de igual forma la localidad y focalidad de la experiencia de aprender y conocer", (Da Costa, 2007, p. 13).

Se deben superar las prácticas educativas basadas en la idea de la universalidad de la infancia, hoy se sabe que cada contexto y realidad personal de la niñez marca su desarrollo, debemos optar por una pedagogía de posibilidades más abiertas que propicien la equidad y justicia social contenida en el significado de la educación. Es necesario explicar la opción de "actores". El ser actores, posiciona a la educadora y al educador como miembros más del proceso en vez de otorgarles el estatus y "poder" por sobre los educandos.

Al respecto Cordero (2005) se refiere a la participación social de la niñez desde la intersubjetividad, entendiendo la intersubjetividad como la comprensión que se comparte en cuanto a propósitos y objetivos comunes, como un proceso que en sí mismo implica intercambio cognitivo, social y emocional. Por su parte Dahlberg, Moss y Pence (2005, p.97) sostienen que "las identidades se construyen y se reconstruyen en el seno de contextos específicos que están siempre abiertos al cambio y en los que el significado de lo que los niños son, podrían ser y deberían ser, no puede quedar establecido de una vez por todas".

Se entiende lo anterior como la necesidad de considerar al infante como un ser involucrado en un contexto, el cual lo influye y del cual él es parte importante, pues es en este donde interactúa con diferentes agentes, que a la vez, le ayudan en su crecimiento, posición que se articula con lo mencionado por Da Costa (2007, p. 9), cuando señala, "cada tipo de infancia está regida por el hacer social en donde tiene lugar".

Se retoma a Dahlberg et al. (2005) quienes señalan que vivir en una sociedad caracterizada por condiciones posmodernas significa que cada niño y cada niña (sic) tienen que ajustarse a un elevado grado de compleji $\neg$ dad y de diversidad, así como a cambios continuos. En una sociedad más estable, la biografía y los conocimientos de los niños venían casi predeterminados, más o menos como los de sus madres y padres. En tales condiciones, podía entenderse la función de la pedagogía de la primera infancia como el hacer posible que los infantes asumieran su identidad verdadera (esencial), y la de la reproducción del conocimiento y los valores culturales, predeterminados antaño por la religión y, más recientemente, por una ciencia y una razón supuestamente objetivas y libres de valores.

\subsection{La educación en los primeros años de vida tiene que ser integral, no únicamente de preparación para la escuela}

La niñez requiere de una educación integral que potencie las dimensiones socioafectiva, psicomotora y cognitiva y que contribuya a favorecer su óptimo desarrollo.

$\mathrm{Al}$ respecto, Jaramillo (2007) señala que una de las tendencias en educación infantil en el mundo moderno es el logro de la formación integral del niño, tal como lo planteó la UNESCO la Comisión Internacional sobre la Educación para el Siglo XXI, donde se explicitaron cuatro dimensiones de aprendizaje humano: aprender a conocer, aprender a hacer, aprender a ser y aprender a vivir juntos.

Es un hecho que en la experiencia con algunos programas educativos dirigidos a la niñez ha prevalecido la idea de formarlos para la escuela, con lo cual se resta al nivel de educación inicial su relevancia, se ha trabajado arduamente en la academización de la experiencia antes que un abordaje integral. Justamente por eso se le ha denominado a este ciclo preescolar, preprimaria.

Para Peralta (2004, p.71) "la educación es un proceso permanente de perfeccionamiento humano que comienza desde el nacimiento". En este sentido, las opciones que ofrece el país deberían entonces, orientarse al desarrollo integral, pero no solo plantearlo por escrito o en los propósitos de los programas y legislación, sino en la práctica, que la oferta se traduzca en acciones tendientes a favorecer el desarrollo integral de las personas en las diferentes dimensiones del ser humano.

Se debe superar el ámbito puramente cognitivista e implementar una formación más integral, involucrando la sensibilidad y los afectos, la realidad de lo comunitario y lo social; además, 
permitir, explorar y familiarizarse con el sentido y significado de las realidades, así como formarse juicios "correctos" sobre sí mismo, sobre el mundo y la sociedad.

Otra arista que se debe considerar cuando se trata de desarrollo y aprendizaje es precisamente el ambiente donde se desenvuelven las personas, así como las relaciones que puedan establecer, al respecto Peralta (2005, p.97) señala:

Se hace necesario comprender que para que los niños y niñas (sic) puedan tener desempeños adecuados para la vida $\mathrm{y}$ aprendizajes de mayor calidad, requieren haberse desarrollado en ambientes saludables e interesantes cognitivamente, donde el afecto y la nutrición adecuada son fundamentales tanto para el desarrollo cerebral como para establecer las bases de la personalidad a través de las relaciones que realiza con los demás y su entorno.

Desde la posición de la autora, la nutrición, los juguetes, las otras personas y un lugar estimulante y acorde con cada edad, hace que las funciones cerebrales mejoren y se potencien. De esa forma, la influencia del apego, el amor y el afecto se convierten en factores más que importantes, en cruciales para esta población, dado que a partir de ahí, formarán su personalidad y ampliarán su desarrollo.

En virtud de estas visiones se considera fundamental que la experiencia de educación y atención de la niñez menor de 3 años se oriente hacia el desarrollo integral que identifica las diferentes dimensiones del ser humano, la salud, lo educativo y a la necesidad de que la persona se desarrolle en esos ámbitos de manera integrada y simultánea. Es decir, sobrepasar realmente la idea de preparar para la escuela, negando así el valor de las experiencias cotidianas en el desarrollo de los niños. Todo un reto para los programas dirigidos a promover el desarrollo integral de la niñez menor de 3 años.

\subsection{Ambientes cotidianos deben promover el desarrollo integral de la niñez}

Las características de la niñez menor de 3 años exigen repensar la orientación de su abordaje; es decir, resulta imprescindible ofrecer ambientes o escenarios que impliquen experiencias que favorezcan la cotidianidad, lo usual en una cultura dada (Chavarría et al., 2007). De ahí la importancia de mantener una actitud por parte del adulto, más de acompañamiento, un ambiente cálido, acogedor, de estar presente, de facilitar en vez de academizar. Un ambiente humano grato, que destaque antes del empleo de estrategias didácticas o materiales específicos, "contextos lo más naturales posibles, desechado la artificialidad excesiva y sin desvincular las experiencias de las instancias habituales" (Peralta 2008, p.45), donde las personas adultas asuman una actitud preocupada, receptiva y facilitadora hacia el desarrollo los niños.

La infancia es muy importante en la vida de las personas, pues tanto el crecimiento como el aprendizaje se dan de manera conjunta, además, es la etapa en la que más rápidamente se aprende. Cada acontecimiento en su vida tiene un gran significado, razón por la cual es un proceso diferente en cada niño donde una de las tareas principales es la autonomía, por lo que deberían implementar acciones por sí mismos y crear sus propias ideas sobre cómo deben suceder ciertas situaciones.

Según Peralta (2008) toda actividad con bebés se debe sustentar siempre en el bienestar integral, para esta pedagoga eso significa que "el niño se sienta cómodo, con personas $<<$ amorosas $>>$, en ambientes conocidos y en situaciones libres de estrés" (p. 43).

En resumen: los niños requieren ser atendidos en ambientes humanos que les provean la oportunidad de desarrollo desde la cotidianidad, en las acciones más simples como el momento de la alimentación, del baño, de jugar libremente, de colaborar de acuerdo con sus posibilidades en el arreglo de los ambientes. Es por ello, que no puede existir una forma única o universal de educarlos o atenderlos, sino más bien, construir 
valores coherentes con actitudes por parte de las personas adultas de aprovechar los momentos compartidos para potenciar su desarrollo, sin caer en el academicismo. Para ello se necesita retomar ciertos espacios como los parques, los paseos familiares, las tertulias con historias y cuentos, las plazas, acomodar y sembrar juntos, disfrutar a la niñez en su máximo esplendor. En ese contexto sociocultural actual, junto con las familias y las personas especialistas en diversas áreas como la educación infantil, debe involucrarse la comunidad entera en sus diversos escenarios para ampliar la riqueza de experiencias y su vinculación con la realidad, todo esto, con la convicción de que la mejor inversión que se puede hacer en amor e inteligencia, es con los niños pequeños. Es imperativo, asumir una práctica más abierta apegada a la cotidianidad, que garantice a la niñez un desarrollo sociocultural pertinente.

\subsection{La atención integral para el desarrollo de la niñez menor de 3 años alude a un trabajo compartido de diferentes especialidades, educación, salud, psicología y nutrición}

La atención integral se refiere al desarrollo humano y a los cuidados que se le brindan a la niñez durante sus primeros años. A partir de ese posicionamiento, tomar acciones que aspiren a fortalecer el desarrollo de los niños menores de 3 años exige el trabajo conjunto desde diferentes especialidades a fin de cubrir y potenciar sus experiencias, desde la salud, para la sobrevivencia de la niñez, prevención y protección de enfermedades; desde la educación, para lograr ambientes de calidad y aprendizaje humano; y desde la nutrición, garantizar los nutrientes necesarios para el óptimo desarrollo de los niños.

Es preciso enfatizar que en todas esas acciones perderían efectividad si la familia no está involucrada en esa lógica hacia el desarrollo integral, pues los estilos de crianza son determinantes, de ahí la importancia del involucramiento consistente y relevante de la familia en los programas para la educación y atención de la niñez ya sean formales o no formales, aspecto fundamental tratándose de bebés, (Arias, 2012) y (Peralta, 2008).

Por otra parte, la educación y la atención de la niñez menor de 3 años debe asumirse para garantizar el desarrollo humano, más que proyectando el beneficio económico de los países. Existen numerosos argumentos de orden social, económico, político y moral para tomar la decisión de invertir en la educación y atención de la niñez menor de 3 años, entre ellos se encuentra que la inversión en políticas para la niñez se recompensa por una parte, en productividad económica a largo plazo y -por otra- en la formación integral que preserva los valores morales y democráticos.

\section{La atención y educación de la niñez menor de 3 años desde la legislación costarricense}

La atención y educación de la niñez menor de 3 años en Costa Rica tiene un importante fundamento en leyes nacionales y convenios internacionales que el Estado ha firmado con diferentes organismos, así como un enfoque de derechos. Su evolución se puede observar cronológicamente con la creación del Patronato nacional de la infancia en 1930, el Código de infancia en 1932, la Ley de adopciones en 1934, la Constitución política de 1949, la Ley de creación del Instituto Mixto de Ayuda Social en 1971, la Ley de desarrollo social y asignaciones familiares en 1974, el Código de la niñez y la adolescencia en 1998 y la Política nacional para la niñez y la adolescencia 2009-2020. Además, el país ha suscrito convenios internacionales a partir de la Declaración de Ginebra sobre los derechos del niño en 1948, la Declaración de los derechos del niño en 1959, el Año internacional de la familia en 1994, la IV Conferencia mundial sobre la mujer en Beijing y los Objetivos del milenio en el 2010. Al respecto, se puede evidenciar un accionar bien intencionado y coherente con los valores establecidos socialmente para este grupo poblacional, sin embargo es importante afirmar que a pesar de ello, la creación o ratificación de la ley no ha garantizado su cumplimiento real en Costa Rica, donde la niñez menor de 3 años se ha quedado al 
descubierto, porque en su accionar el Estado no ha brindado una respuesta efectiva para su educación integral.

Como complemento del marco legal se creó una serie de programas cuyos propósitos fueron -entre otros- mejorar la situación nutricional de la población materno infantil que vivía en condición de pobreza (CEN-1950); además, tratando de dar respuesta a la crisis económica y social de la década de 1980 con opciones de cuido para que las madres trabajaran fuera del hogar y así ofrecer tanto la atención como la educación de este grupo, dentro del espacio formal y del no formal. En el ámbito no formal se cuenta: CEN-CINAI, que atiende a la niñez mayor de 2 años y menor de 7 , los Hogares comunitarios, que por su parte, atienden a los niños menores de 7 años. Por otra parte, están los centros infantiles privados, que al igual que los centros infantiles de instituciones autónomas atienden población menor de 5 años y, por último, está la Red nacional de cuido que ofrece atención a los niños hasta los 12 años de edad, en condición de vulnerabilidad social, pero con posibilidades de aportar económicamente para el cuido de sus hijas e hijos.

Dentro del ámbito de lo formal está el Ciclo o Programa materno infantil administrado y operacionalizado por el Ministerio de Educación Pública, el cual atiende a niños desde 4 años y 6 meses hasta 5 años y 6 meses. Como se observa, el Estado costarricense ha creado numerosos programas dirigidos a la niñez; sin embargo, es imperativo resaltar que dichos programas, exceptuando algunos centros infantiles privados, los de instituciones autónomas, así como el del Ministerio de Educación, enfocan su atención en la niñez en condición de vulnerabilidad, lo cual es relevante aunque representa una restricción en su cobertura, puesto que deja desprovista a la niñez que no está bajo la línea de pobreza y que no por ello, deja de necesitar atención y educación integral.

\section{Propuestas programáticas para la atención actual de la niñez menor de 3 años en Costa Rica: visión de los procesos de formación y sus participantes}

En esta investigación se analizaron diferentes documentos como los siguientes: la Guía curricular para el desarrollo integral para el niño menor de 6 años (1982), la serie Creciendo con amor, el Programa materno infantil del Ministerio de Educación Pública y las Guías del Ministerio de Salud elaboradas en el año 2011. Del análisis respectivo se desprende que los lineamientos teóricos presentes, tanto en la Guía del año 1982, como en la Serie Creciendo con amor, el planteamiento corresponde a un enfoque más positivista, mientras que en el Programa Materno Infantil y la Guía de Educación Inicial 2011, su enfoque es Holista, es decir, favorece el desarrollo integral de la niñez que implica contar con una apropiada nutrición, atención en salud, amor, estimulación psicosocial e interacciones significativas con sus padres y con otros adultos que ejercen algún tipo de influencia en su proceso de crianza, estos enfoques responden a las corrientes teóricas vigentes en cada época. Al respecto, cabe señalar que el programa Materno Infantil, a pesar de que en sus planteamientos abarca a la niñez desde los primeros meses de edad, el Estado atiende únicamente a partir de los 4 años y 6 meses y al igual que los demás, deja por fuera a los menores de 3 años.

En forma general, se podría argumentar que estos documentos no están bien posicionados paradigmáticamente, salvo alguna excepción. En este sentido, el que sí responde a un paradigma holista, no garantiza que su aplicación resulte coherente con esos planteamientos. Cabe recordar que un paradigma holista vendría a favorecer el desarrollo de las capacidades y los talentos particulares, a estimular la disciplina y el amor mutuo y a considerar al docente como un recurso y facilitador que responde al potencial de cada niño, así como a invitar a la niñez a ser aprendices participando activamente. 
4. Hallazgos en torno a las guías programáticas para la niñez menor de 3 años en Costa Rica

Respecto de los aspectos esenciales que permean la visión de los procesos de formación y sus participantes en los programas principales para la atención actual de la niñez menor de 3 años en Costa Rica se presentan el análisis de las propuestas programáticas destinadas a la educación y la atención de la niñez menor de 3 años; se evidencia en todas un posicionamiento teórico que apuesta por lograr un desarrollo óptimo de la niñez, para ello:

Tanto la Guía Curricular (1982), la Serie Creciendo con Amor, así como la Guía para la elaboración de planes de estimulación del Ministerio de Salud 2011, están posicionadas en un enfoque teórico cognitivo conductual que proponen según Chavarría et al. (2007, p.33) que "los seres pasan por etapas predecibles universales bio-psico-socioemocionales". Por contraste el Programa Materno Infantil y la Guía de educación inicial 2011 procuran su accionar en principios del desarrollo infantil el primero y en derechos de la niñez la segunda, que responden al enfoque humanista u holista del desarrollo infantil.

La Guía Curricular (1982), está organizada desde un posicionamiento conductual positivista que asume el desarrollo por etapas obviando que el ser humano posee formas diversas para obtener el conocimiento. También enfatiza en los resultados del aprendizaje, en el caso particular, en las conductas que la niñez debe manifestar al final de cada fase. No es casual por tanto que la Guía se denomina de estimulación, pues el propósito es estimular, hoy se enfatiza en ofrecer oportunidades para el desarrollo de la persona. (Peralta 2002, 2005, 2006 2007)

La Serie Creciendo con Amor al igual que la Guía Curricular (1982), se organiza por áreas de desarrollo mediante un planteamiento Piagetiano. Plantea la serie un visión estereotipada de las mujeres cuando se les endosa la responsabilidad del cuido de la niñez dadas sus condiciones de ternura, sensibilidad y protección, negando así, ese derecho a los varones y perpetuando prácticas sexistas en nuestra sociedad.

El Programa del MEP, se fundamenta en el desarrollo integral, cuyo propósito es la preparación para la vida.

La Guía para la Educación Inicial 2011 por su parte, corresponde a un modelo de educación infantil de marco abierto que integra teorías holistas e integradoras.

Llama la atención la Guía para la elaboración de planes de estimulación para la promoción del desarrollo infantil de niños y niñas de 1 a 6 años y 11 meses, pues asume un posicionamiento teórico por áreas de desarrollo, además propone acciones para superar el "rezago" de los niños en su desarrollo. Una contradicción que hace frágil la propuesta para esa población en el sentido de que lo "evolucionado" que nos evidencia los avances en esa materia, regrese a una visión de desarrollo por etapas, cuando muy bien la Guía de Educación Inicial (2011), se planteó que en adelante el trabajo arduo sería para "crear significados a través de oportunidades $\mathrm{y}$ actividades culturalmente pertinentes que permitan a las niñas y los niños aprender con significado". García (2011, p.40)

\section{Población beneficiaria}

- El programa Materno Infantil, aunque involucra a los niños desde los 2 meses, no los atiende en lo formal, sino que atiende a partir de los cuatro y medio años. La guía curricular del MS (1982) incluye a los niños de días de nacidos hasta los 6 años. No obstante el programa de CEN 
y CINAI los atiende a partir de los 2 años, y la serie Creciendo con Amor involucra a la niñez de meses hasta los 7 años y en algunos Hogares Comunitarios se atienden niños de 6 meses en adelante. También es importante destacar que el programa Materno Infantil asume a la población en general, sin distingos por su condición económica o social, en opinión de la investigadora, una propuesta también pública como las anteriores guías, pero con una cobertura más equitativa. Las guías del Ministerio de Salud 2011, también incluyen a la niñez de 1 a 6 años y 11 meses, interesante, pues la edades de 0 a 11 meses y 29 días según estas guías ¿quién las atiende?

Es necesario indicar que tanto las propuestas programáticas del Ministerio de Salud y la serie Creciendo con Amor, mantienen su interés en la población de niños más desposeídos o en estado de desnutrición, además de procurar espacios de cuido para que a las familias se les posibilite trabajar, tal como lo indica la Guía de Educación Inicial "da aporte a un perfil particular de promotor social institucional" García (2011, p.9).

\section{Principios orientadores}

- En relación con los principios orientadores las guías programáticas comparten el desarrollo integral, no obstante, la Guía Curricular (1982) y la Serie Creciendo con Amor se estructuran por áreas de desarrollo mientras que el Programa Materno Infantil, y la Guía de educación Inicial del Ministerio de Salud 2011, asumen un abordaje más holista de los principios curriculares y en los derechos de la niñez. En tanto la Guía 2011 para la elaboración de planes de estimulación del Ministerio de Salud parte de la premisa del rezago en el desarrollo como iniciativa de creación de planes.

\section{Concepción de niño}

- La concepción niño tanto en la Guía (1982) como en la Serie Creciendo con Amor y la Guía para la elaboración de planes de estimulación 2011, son seres pasivos que reciben estímulos para su desarrollo en tanto en el Programa Materno Infantil, las niñas y los niños son actores de su propio desarrollo en interacción con el contexto sociocultural por ello el personal encargado involucra a la familia y a la comunidad. Estas últimas, son propuestas coincidentes con una postura filosófica personalista que concibe a la niño como "persona y como tal ha iniciado su proyecto de existencia desde que nace. Por tanto, es sujeto de derechos y deberes" Peralta (2006, p.274).

\section{Relación con la familia}

- Tanto en la Guía 1982 como en la Serie Creciendo con Amor, se pensó en la estructura de la familia tradicional papá, mamá e hijas e hijos; mientras en el Programa Materno y en las Guía del Ministerio de Salud del 2011, se enfatiza en la importancia de la familia y de las implicaciones culturales y de desarrollo que tiene el involucramiento de las familias en las acciones que se emprendan con la niñez. Es una visión más de participación de las familias, que como lo indica Peralta (2006, p.53) "los primeros, permanentes y por lo tanto siempre válidos educadores son la familia [...]poseen un conjunto de normas y prácticas de crianza y de vida en definitiva que, [...] los hace ser un factor educativo esencial por valorizar".

\section{Actividades propuestas}

- En las actividades sugeridas en la Guía Curricular (1982), se evidencia una clara intención de preparar para la escuela y algunas de las actividades proyectan estereotipos respecto del aprendizaje de los niños como el caso de los periodos cortos de concentración, en el caso de la serie Creciendo con Amor aún cuando no se expresa el término actividad se sugiere preparar al niño para actividades de tipo académico. En la Guía para la elaboración de planes de estimulación del Ministerio de Salud 2011, las actividades representan conductas que los niños y las niñas deben realizar con base en una evaluación que determina "los rezagos" de cada uno de ellos. Por su parte, en el Programa Materno Infantil como en la Guía de educación Inicial 2011, las actividades se expresan como acciones por parte de la niñez, seres activos en desarrollo en interacción con sus pares y otras personas adultas. 


\section{Evaluación}

- La evaluación propuesta en la Guía Curricular (1982) y la Guía para la elaboración de planes de estimulación 2011, responden al planteamiento de objetivos conductuales y se proponen determinar el desarrollo de los niños a partir de la Escala EDIN, por su parte la serie Creciendo con Amor no incluye evaluación, mientras el Programa Materno Infantil y la Guía de educación inicial 2011, hacen un planteamiento sistemático como proceso indispensable de la planificación.

\section{Papel del adulto}

- En relación con el desempeño de la persona adulta que atiende a la niñez en la Guía Curricular 1982 se plantea que la persona responsable de aplicarla, posea conocimientos mínimos sobre el desarrollo infantil, en tanto para la serie Creciendo con Amor, son personas sin estudios superiores algunas veces ni educación media, la mayoría en condición de amas de casa quienes los atienden; en el Programa Materno se presenta un perfil con rasgos que debe poseer la persona profesional en ese ámbito. Quien aplica los planes de estimulación recomendados en la Guía para la elaboración de éstos 2011, es el o la Asistente de Salud bajo la supervisión de la persona profesional designada por la Dirección de Servicios correspondientes. La Guía de Educación Inicial del Ministerio de Salud 2011, visualiza a la persona adulta como aquella que "educa mediando entre la cultura, la naturaleza y el niño y niña: Programa actividades y presenta ambientes para educar para la vida" García (2011, p.25).

- Es necesario señalar que las Guías fueron elaboradas por instituciones del Estado con propósitos diferentes, de ahí la diversidad de enfoques, sin embargo la niñez requiere de programas que aseguren su atención integral, programas de calidad porque, como bien lo indica Peralta (2006) los programas de baja calidad reproducen el círculo de pobreza.
- Considerando tanto los años en los cuales fueron creadas las Guías programáticas, como su contexto, el Programa Materno Infantil (2000) y la Guía para la Educación Inicial 2011 presentan un avance importante en cuanto a lineamientos teóricos, abordaje, estructura curricular y actores en comparación con la Guía curricular (1982) la serie Creciendo con Amor, y la Guía para la elaboración de planes de estimulación para la promoción del desarrollo del desarrollo infantil de niños y niñas de 1 a 6 años y 11 meses (2011). No obstante, no se ha realizado por parte del Ministerio de Educación una evaluación de ese programa que valore su impacto en términos de desarrollo para los niños. En la actualidad el Departamento de Educación Preescolar del Ministerio de Educación está en el proceso de capacitación a las docentes del nivel preescolar respecto del nuevo programa de estudios y de la Guía Docente del Programa de Estudios que involucra tanto el ciclo Materno Infantil como el de Transición. Es importante señalar que dicho programa 2014 no fue objeto de análisis en la investigación citada.

- Por su parte el Ministerio de Salud actualizó la Guía de Estimulación para el Desarrollo Integral del niño menor de 6 años. En general, respecto de la Guía de educación inicial 2011, se puede indicar que es un recurso muy valioso, pues no existía en el país una propuesta curricular tan actualizada y apegada a un enfoque humanista de la acción educativa para la atención de la niñez en contraste con la Guía para la elaboración de planes de estimulación para la promoción del desarrollo infantil de niños y niñas de 1 a 6 años y 11 meses, analizada anteriormente. 


\section{Reflexión final}

A manera de conclusión se puede indicar que a pesar de los diferentes convenios, normativas, leyes y reglamentos existentes tanto en el plano internacional como en el nacional en relación con la atención y educación de la niñez menor de 3 años, en Costa Rica, ningún documento ha garantizado su aplicación efectiva, ni una respuesta coherente que involucre a este grupo poblacional. El Estado costarricense invierte de manera escaza en esta población; es decir, no representa una de sus prioridades, desde lo formal, en consecuencia la oferta de educación y atención de la niñez menor de 3 años en el país es insuficiente. Por otra parte, la política pública destinada a la niñez en Costa Rica se basa más en la protección de los derechos de la niñez y presenta vacíos así como ausencias significativas como el apoyo a los grupos familiares, la importancia del apego seguro y la estimulación psicosocial del niño.

\section{Referencias}

Arce, M., Simón, C.\& Villalobos, G. (1993). Agua Miel. San José. Editorama.

Arce, M., Simón, C.\& Villalobos, G. (1993). 1-2Agua, Tierra y Sol. San José. Editorama.

Arce, M., Simón, C.\& Villalobos, G. (1993). Brinca La Tablita. San José. Editorama.

Arce, M., Simón, C.\& Villalobos, G. (1993). Colorín Colorado. San José. Editorama.

Arce, M., Simón, C.\& Villalobos, G. (1993). Dame la Mano. San José. Editorama.

Arce, M., Simón, C.\& Villalobos, G. (1994). Los Niños y las Niñas son así. San José. Editorama.

Arce, M., Simón, C.\& Villalobos, G. (1993). Mi mundo Verde y Azul. San José. Editorama.
Arce, M., Simón, C.\& Villalobos, G. (1993). Trabajando Juntos. San José. Editorama.

Arias, G. (2012). La familia, la educación y el desarrollo infantil. Universidad de La Habana. La Habana: Cuba.

Calvo, A., Campos, S., Mata, J., Morera, A., Naranjo, T., Vargas, K. (2012). Un análisis histórico crítico de la política social de cuido para la niñez en Costa Rica. Memoria del Seminario de Graduación para optar por el grado de Licenciatura en Trabajo Social, Universidad de Costa Rica.

Cárdenas, H. (2014). Propuesta de lineamientos para la educación y atención integral para el desarrollo de la niñez menor de 3 años en Costa Rica. Tesis para optar por el grado de Doctorado de la Universidad Estatal a Distancia, Costa Rica.

Chavarría, G., Orozco, C., Ovares,M., Obando, M., Rivera, M. (2007). Problematización en torno a una educación inicial holista y crítica. (Avances de Investigación) Costa Rica: Instituto de Investigaciones Psicológicas.

Chavarría, M. (2012). Historiando a Montessori: desde el feminismo y socialismo utópico hacia su compromiso como pionero del holismo. Actualidades investigativas en educación. 12(3), 1-33.

Constitución política de Costa Rica. Recuperado de http://www.tramites.go.cr/manual/espanol/ legislacion/ConstitucionPolitica.pdf.

Da Costa M. (2007). La infancia desde una perspectiva post-estructural y transformaciones que supone para la educación pre-escolar. Conferencia presentada en el Congreso Internacional Repensando la Educación Parvularia en América Latina. Universidad Central de Chile, Santiago, Chile. 
García, G., Pérz, O., Poltronieri, P. (2011). Guía de Educación Inicial "Promoción de la salud en torno al eje de la infancia”. San José, Costa Rica.Ministerio de Salud.

Goldschmied, E. y Jackson, S. (2000). La educación infantil de 0 a 3 años. Madrid: Morata.

Ministerio de Educación Pública. (2004). Programa de Estudio Ciclo Materno Infantil Educación Preescolar. 1 ed. San José: MEP.

Ministerio de Salud Pública. (1982). Guía curricular para la estimulación del Desarrollo Integral del Niño menor de 6 años. San José, Costa Rica: CONET (Consejo Operativo Nacional para la Estimulación Temprana).

Myers, R. (1995). La Educación Preescolar en América Latina: el estado de la práctica. Programa de Promoción de la Reforma Educativa en América Latina y el Caribe Partnership for Educational Revitalization in the Americans.

Organización Internacional del Trabajo (OIT) (2012). Un buen comienzo: La educación y los educadores de la primera infancia. Informe para el debate en el Foro de diálogo mundial sobre las condiciones del personal de la educación de la primera infancia. Ginebra, 2012.

PANI-UNICEF. (2009). Política Nacional para la Niñez y la Adolescencia Costa Rica 2009-2021. San José, Costa Rica.

PANI, (2013). Informe evaluación anual metas Plan Operativo 2012. Costa Rica: PANI.

PANI. (1998). Código de la niñez y la Adolescencia. San José, Costa Rica.

Peralta, V. (2004). "Comenzar fuerte y oportunamente". Fundamentos para una educación desde el nacimiento. Revista Perspectivas. Chile, Núm. 18 pp 25-37.
Peralta, V. (2005). Nacidos para ser y aprender. Buenos Aires, Argentina: Infantojuvenil.

Peralta, V. (2008). El Derecho de los más pequeños a una pedagogía de las oportunidades en el siglo XXI. Revista Iberoamericana de Educación Núm. 47 pp. 33-47.

UNICEF. (2001). II Estado de los Derechos de la Niñez y la Adolescencia en Costa Rica. Universidad de costa Rica. San José: Costa Rica. Recuperado en www.unicef.org.

Wernicke, C. (1994). Educación Holística y Pedagogía Montessori. Educación Hoy. No. 10 pp.1-11. Recuperado de http://cepcadiz. com/materiales/ARC_2_29_20.pdf 